\title{
Sensory deprivation in spinal cord injury - an essay
}

\author{
Mark W Crossman \\ Chief Resident, Division of Physical Medicine and Rehabilitation, The University of British Columbia, Vancouver, \\ Canada
}

\begin{abstract}
Due to altered or absent sensation below the level of the lesion, and varying degrees of preserved motor function, the spinal cord patient is limited in his/her ability to increase sensory feedback via interaction with the environment. The sequelae of this in the acute spinal cord patient could be likened to a state of sensory deprivation. Drawing inferences from the sensory deprivation literature of the 1950s and 1960s, in which university students volunteered to be placed in sensory deprivation chambers, is far from accurate. However, the research stimulates thought into the combined effects of immobilization, social isolation, and sensory deprivation-all of which the acute spinal cord patient is likely to experience. This state of sensory deprivation appears to lessen with time, regardless of the persistence of the impairment. The essay hypothesizes that neuroadaptive processes occur and proposes the use of sensory stimulation, particularly in the spinal intensive care unit, to facilitate this adaptation.
\end{abstract}

Keywords: spinal cord injury; sensory deprivation; auditory vigilance

It was completely by accident that I started to consider the concept of sensory deprivation in the spinal cord injured population. While doing a spinal rotation as a physiatry resident at the GF Strong Rehabilitation Center in Vancouver, Canada, I noticed a nurse walk by holding a round plastic scrub brush, primarily used to massage shampoo into the scalp. The nurse described how wonderful the scalp sensation was for the patients and that they requested it often, not just when being shampooed. These patients were 'high lesion', classified as C4 or rostral in our center.

The 'scrub brush incident' raised many questions about what it is like to live with a spinal cord injury. Was the extreme tactile pleasure experienced by this group of tetraplegic patients the result of the sensory receptors being 'starved' for any tactile stimulation at all and thus being ultra-sensitive? Could this be analogous to the up-regulation of receptors that occurs when a chemical substance is in short supply? Alternatively, is it that the spinal cord patient just attends to these stimuli to a greater degree than do people with intact spinal cords, due to the psychological need?

The spinal cord patient is in a state where sensation is decreased to varying degrees below the level of injury. In addition, motor function is also either decreased or completely absent below the lesion. This robs the patient of proprioceptive feedback and limits his/her ability to increase sensory feedback via

Correspondence: Mark W Crossman BSc, MD. BC Rehab, 4255 Laurel Street, Vancouver, B.C. Canada V52 2G9 interaction with the environment. The sequelae of this in the acute spinal cord patient could be likened to a state of sensory deprivation. This state appears to lessen with time, regardless of the persistence of the impairment. An understanding of this concept, and whether neuroadaptive processes occur, is important to be able to devise potential treatment strategies engaging sensory stimulation to facilitate adaptation and to improve quality of life.

Sensory deprivation is defined as an 'absolute reduction of sensory input' whereas perceptual deprivation is a 'distortion of the patterns of sensory stimulation rather than reduction in the absolute amount'. Both types of experiences are pertinent to the spinal cord patient. (For the most part, I will be using the term 'sensory deprivation' to refer to either experience).

Interest in the area of sensory deprivation was stimulated by reports of explorers, astronauts, and prisoner's of war, all of whom endured sensorydeprived environments. ${ }^{2}$ Surprisingly, during the fad of experimentation in sensory deprivation in the 1950s and 1960s, few considered studying the spinal cord patient, a 'natural experiment' in sensory deprivation. We are forced, therefore, to look at the research as it stands, mostly using healthy university students in various situations where this state was artificially created in a laboratory. This is not to say that we cannot make cautious inferences back to the spinal cord patient.

Numerous negative effects have been associated with sensory deprivation. These have consisted of the 
following: (1) poor tolerance for the environment; ${ }^{3}$ (2) psychomotor retardation and depression; ${ }^{3}$ (3) perceptual changes such as fantasies, delusions, or hallucinations; ${ }^{3}$ (4) intellectual and cognitive impairments; ${ }^{3}$ (5) physiological changes such as changes in galvanic skin response, ${ }^{4 a}$ and an increased proportion of slow waves in the parieto-occipital cortex on EEG; ${ }^{4 \mathrm{~b}-\mathrm{d}}$ and (6) chemical changes such as increased urinary levels of epinephrine and norepinephrine ${ }^{4 \mathrm{e}}$ and increases in plasma TSH and urinary 17-ketosteroids and 17ketogenic steroids. ${ }^{4 \mathrm{f}} \mathrm{I}$ will not even attempt to review all the sensory deprivation literature, as there is an enormous body of research. The literature is also difficult to interpret at times due to confounding variables, various experimental designs, the use of different psychometric measures, and lack of standard terminology. 5,6

I have chosen to briefly review the literature looking at particular sub-themes of the spinal cord injury experience. With spinal cord injury, the absence of tactile and proprioceptive sensation does not occur in isolation. Along with this is the effect of immobilization itself and, at times, the devastating effects of social isolation. Many of the early experiments have these overlapping and confounding variables, but subsequent research tries to investigate the sub-themes.

The effects of immobilization were evaluated with 96 male university students who volunteered to lie for seven days in an immobiliziation box consisting of a head-holding device, a foam rubber lining, and straps to hold them immobile. ${ }^{7}$ One-third of the subjects were randomly assigned to each of three groups, one experimental and two control. Of the control groups, one was ambulatory and the other recumbent but not immobilized. The experiments tried to still expose all groups to environmental stimuli. Performance on a battery of psychological tests before and during the experiment was compared for each group.

Overall, the experimental group performed more poorly than either of the control groups. ${ }^{7}$ This included such tests as recall, space relations (selecting portions of figures that might form a design), and verbal fluency. There was also a poorer performance in the recumbent than in the ambulatory controls. ${ }^{7}$ The authors concluded that this indicated that there was a combined effect of recumbency and immobilization on reducing performance on intellectual and perceptualmotor tasks. ${ }^{7}$ For spinal cord patients, the combined effects are seen, again, mainly during the acute stages, with the decreasing periods of recumbency as stabilization from their medical condition and postural hypotension allows.

Another group looked at whether social stimulation decreases the effects of immobilization and sensory deprivation and found that social contact does not eliminate the negative effects of sensory deprivation, but it does lessen them. ${ }^{8}$

The theory behind many of the negative effects of sensory deprivation is entitled the 'deafferentation theory'. ${ }^{9}$ This theory assumes that organisms act on their environment in a stimulus-seeking role so as to arrive at the optimal level of arousal. ${ }^{00}$ That such is true would predict that organisms under situations of sensory deprivation would be in a state of relative decreased cortical arousal. ${ }^{2}$ Lilly ${ }^{11}$ asked, 'Freed of normal efferent and afferent activities, does the brain soon become that of coma or sleep, or is there some inherent mechanism which keeps it going, a pacemaker of the awake type of activity?'

The neuroanatomical substrate responsible for arousal appears to be the reticular activating system (RAS). ${ }^{12,13}$ Most stimuli are projected to the brain through this system. There are also projections of some ascending fibers to the limbic system that would partially account for the emotional responses to sensory stimuli (along with the meaning of the stimulus to the individual as determined by memories of past experiences). ${ }^{12,13}$ It is presumed that this RAS primes the individual with a level of arousal necessary for normal emotion, perception, and learning. In addition, we think (from the experiments on sensory deprivation and perceptual deprivation) that it is not only the quantity of stimulation that is important, but also the quality, as represented by the patterning of stimuli with appropriate degrees of variation.

In the spinal cord patient, ascending spinoreticular fibers would, depending on the completeness of the lesion, be interrupted. This leads one to question if there are EEG changes, reflecting altered cortical arousal, in the spinal cord patient. One study ${ }^{14}$ looked at 14 high cervical patients, divided into these two groups: one of early spinal cord injury (eight patients), and one of late spinal cord injury (six patients). ('Late' was defined as injury of at least two years ago). The patients were allowed to pursue normal activities while their EEG was being monitored for a $19 \mathrm{~h}$ period on two separate occasions. Results showed a distortion in the relative proportions of time spent in particular phases of sleep. ${ }^{14}$ The proportion of time spent in light sleep was $78 \%$ in the late spinal injury group instead of the usual $30-$ $40 \% .{ }^{14}$ There was a decrease in intermediate and deep sleep, seen to a greater degree in the late than early spinal cord injury group. ${ }^{14}$ However, there was a large degree of variation in phases of sleep, even within the same individual. ${ }^{14}$ Unfortunately, it is difficult to form any firm conclusions on this data due to the small number of subjects, the mixture of patients with complete and incomplete lesions, and the lack of any statistical analysis. In addition, the early spinal cord injured patients were most likely turned every $2 \mathrm{~h}$ to prevent decubiti, thereby possibly further skewing the results.

The same investigators ${ }^{14}$ also did EEG's on three patients with thoracic injuries. The EEG's of this group more closely resembled normal sleep patterns. ${ }^{14}$ Again, this can only be considered as an observation. One would also have to control for medication effects 
that might influence results. There are, unfortunately, no recent studies on EEG changes in the paraplegic or tetraplegic patient using proper methodology.

From the literature it is obvious that we do not completely understand the effects of spinal cord transection on the EEG. If we still agree with the deafferentation theory, then the decreased input through the RAS should decrease cortical arousal and therefore decrease performance. Therefore, spinal cord patients should perform more poorly on tasks requiring concentration.

Hester $^{15}$ compared thoracic and cervical spinal patients with normal controls, under increasing levels of motivation, on a verbal coding and reaction time task. Different levels of motivation were used because the theory, termed 'the activation theory of motivation $^{16 a, b, c}$ states that higher levels of motivation should lead to greater cortical arousal and thus improved performance. The results showed no differences in either task between the spinal cord patients and the controls. ${ }^{15}$ They did, as expected, improve in performance with increased motivation. ${ }^{15}$ However, it is hard to separate this from practice effects alone.

The most recent research on performance in the spinal cord patient is by Richards et al..$^{2,17}$ Their criticism of Hester's study was that no difference was seen between the two groups because the task had too great an arousal level which might have masked the deficits among the spinal cord group. ${ }^{2}$ Presumably, the task stimulated the depressed cortical activation of the spinal cord patients above a certain threshold so that they performed as well as the controls.

The first study ${ }^{2}$ used a task considered very monotonous and unlikely to lead to a high arousal level-an auditory vigilance task. The subjects were to respond with the word 'tone' when they heard the 1000 cycle tone which was randomly presented every few seconds over a span of $45 \mathrm{~min}$. EEG data was also obtained at baseline and during the testing. They defined decreased vigilance as a replacement of the alpha wave form by slow wave activity. The subjects included 15 tetraplegic inpatients, seven paraplegic inpatients, four tetraplegic outpatients, and 16 noninjured controls. There was no mention of the level of spinal cord injury, the degree of completeness, or how many subjects were excluded and for what reasons. In addition, controls were volunteers from the hospital staff and might represent a biased sample. The experimental groups were also very small and not of equal sizes. Furthermore, it was not explained why there was no paraplegic outpatient group.

Given the numerous problems with study design, the results showed a decrease in performance and alpha quantity over time for both the experimental and control groups. ${ }^{2}$ The tetraplegic outpatient group consistently outperformed all other groups. ${ }^{2}$ In addition, the subjects more recently injured (inpatients) performed worse than the more remotely injured and the controls. ${ }^{2}$ The recently-injured also showed a greater decrease in alpha activity than the other groups. $^{2}$ (The authors maintain it was not a medication effect). ${ }^{2}$

It is not surprising that the more recently injured patients fatigued more easily and showed greater decrease in performance on the auditory vigilance task. That is to be expected, due to a shorter period of time to adapt to the injury mentally and physically, along with symptoms such as pain that might interfere with vigilance on a task. What the authors found surprising was that the tetraplegic outpatient group performed better than the controls. ${ }^{2}$ The result, along with Hester's study, ${ }^{15}$ was in direct contradistinction to the deafferentation theory of cortical arousal. There are three possible explanations as follows: (1) tetraplegic patients with nonrecent injuries were more motivated to do the task; (2) adaptive processes had occurred and the patients, not receiving interfering sensory input from other modalities, were able to attend to tasks such as auditory vigilance to a greater degree; and (3) a combination of the above.

The same authors repeated the above experiment with a slightly different task. ${ }^{17}$ It resulted in, again, better performance in the tetraplegic patients than in the controls. ${ }^{17}$ Once more, it is difficult to come to any firm conclusions, as motivational factors in this experiment may have differed because the tetraplegic group was paid to participate whereas the controls were non-paid volunteers.

One might postulate that ascending influences to the RAS are not the only ones of import to affect alertness. Possibly afferent impulses from the face and neck alone are sufficient to maintain activation and cortical stimulation. ${ }^{18 \mathrm{a}}$ We know that afferent inputs are supplemented by projections from the parietal cortex, including the general somatosensory area, to the parvicellular reticular nucleus and then to the central group of nuclei. ${ }^{12}$ Hilgard and Bower ${ }^{18 b}$ stated that possibly 'we can think ourselves into an excited state and thinking can keep us awake'.

There is also evidence in the sensory deprivation literature of increased auditory vigilance in the deprived subjects relative to the controls. ${ }^{6}$ Perhaps decreased sensory input in one modality is offset by increased acuity in another or in other areas of the body. The spinal cord patient could be described as being 'starved' for sensation. A 'cocoon syndrome' has been described in which the patient with a spinal cord injury pulls the covers over his head at night to increase temperature and comfort. ${ }^{19} \mathrm{~A}$ tetraplegic employee at GF Strong told me this gave him a great feeling of security and relaxation to feel the warmth of his own breath on his face. Another tetraplegic person mentioned that in the early days after his injury he craved having a light above his head so he could feel the warmth of the light-bulb on his forehead.

The intensity of the experience of sensory deprivation in the spinal cord population is best described in 
literature on the intensive care unit (ICU) experience. In the ICU environment the spinal cord patient has numerous senses deprived of usual sensory input. Visual stimuli are restricted due to being in a recumbent position which forces one to look at the ceiling. ${ }^{20}$ Also, wearing a halo eliminates the temporal portions of the visual field. ${ }^{20}$ The patterning of auditory stimuli may be reduced due to the monotonous sounds of machines, alarms, and ventilators. ${ }^{20}$ Olfactory sense might be decreased from nasal congestion due to the altered physiology of spinal shock. ${ }^{20}$ Gustatory sense also might be affected due to the initial with-holding of oral intake due to paralytic ileus. ${ }^{20}$ Finally, the decreased tactile and proprioceptive sensation that comes from the spinal cord injury itself completes the picture of strict multi-modality sensory deprivation. This, of course, occurs along with possible social isolation due to restricted numbers of visitors allowed in most ICU's, and decreased interaction with the environment imposed upon the patient by decreased mobility. In addition, the stresses of sleep deprivation are superimposed upon the patient. It is no wonder that we talk of "ICU psychosis'.

One group ${ }^{20}$ investigated perceptual deprivation in the acute spinal cord patient by having all spinal cord injury cases in the ICU over a four year period observed by a clinical psychologist. The psychologist tabulated all behavioral disturbances, defined as episodes of disturbed perception of any sensory modality by the patient not attributable (according to the medical staff) to biochemical or physiological conditions.

Out of 187 patients admitted, 12.3\% exhibited disturbed behaviour. ${ }^{20}$ There were a reduced number of family members available to visit this group and a further geographical distance from the family home to the hospital than for those patients who did not have disturbed behavior. $^{20}$ (This was statistically significant). There was some indication of a greater frequency of disturbed behavior in the higher level injuries, but there were no statistics done on this. Furthermore, behavioral disturbances were poorly defined and often depended on the patient's report. There was also no mention of screening for past psychiatric history or drug abuse. Nevertheless, we can likely minimize the negative effects of sensory deprivation during the acute phase of spinal cord injury by increasing human contact with significant others.

There has not been much research on sensory deprivation in the acute spinal cord injured patient. Most research has focused on the eye surgery patient who has his eyes patched, the cardiac ICU patient, or the immobilized orthopedic patient. ${ }^{5}$ For the eyepatched patients experiencing perceptual and thought disturbances, reassurance, orientation, and explanation from the nursing staff seemed to be the most helpful. $^{21 \text { a }}$ There is also little research done to prove the effectiveness of any particular measure in helping the sensory deprived patient. Simple things such as positioning patients near a window in the ICU have been shown to decrease delirium in surgical patients. $^{21 \mathrm{~b}}$ It is also suggested that sensory overload can produce effects similar to sensory deprivation. ${ }^{22}$ Thus, other simple measures such as positioning the intermittent positive pressure breathing machine with the outflow valve facing away from the patient might be helpful by reducing the decibel level. ${ }^{5}$

There is also some evidence that exercise might decrease the negative effects of sensory deprivation. Zubek $^{23}$ found fewer behavioral impairments, fewer hallucinations, and a lesser degree of decrease in occipital lobe EEG frequency, in exercising compared with non-exercising subjects undergoing sensory deprivation in an isolation chamber. One wonders what effects physiotherapy has on the spinal cord patient in lessening the effects of sensory deprivation. Some physiotherapists recommend the use of a large therapeutic ball on which the patient is placed and rocked, stimulating his vestibular system. ${ }^{24}$ The vestibular system does feed into the RAS, so such a concept is viable. However, it is yet to be subjected to any form of experimentation.

In this review, I was unable to determine whether there are not only motivational and psychological adaptive processes occurring, but whether neurophysiological changes occur as well. Such changes might occur in spinal cord patients with phantom body pain. Melzack and Loeser ${ }^{25}$ proposed a pattern-generating mechanism in which the abnormal firing demonstrated in the area of the cord just rostral to the lesion likely projects to the brain, imprinting as a painful experience. They hypothesized that, in most paraplegic individuals, the abnormal bursting either stops or is blocked by descending inhibitory influences with the passage of time. ${ }^{25} \mathrm{We}$ also know there is evidence of neuronal plasticity in the animal kingdom allowing compensation by one sensory modality when another is hampered. ${ }^{26,27}$

It would be interesting to do positron emission tomographic (PET) scanning on spinal cord patients compared to able-bodied controls with each group receiving some sensory stimulation. The spinal cord patient might have either a greater metabolic activity in the corresponding somatosensory cortical region, or possibly have compensatory increases in other areas of the brain not normally activated by such a stimulus. Unfortunately, such a study might be difficult as there as problems in determining a stable and reproducible resting state for the brain. 28

The important thing to remember, one patient told me, is that 'we are all different'. Certainly, one patient will react to a spinal cord injury one way, and another patient in a completely different manner. In addition, the level of spinal cord injury is important, with a much greater degree of immobility and sensory deprivation in those who are tetraplegic than in those who are paraplegic. Furthermore, the degree of completeness is crucial, although even spinal cord patients with complete lesions have some degree of sensation below 
the level of injury. The sensation is altered, but after a period of time they learn what various sensations mean, such as an imminent urinary tract infection. One tetraplegic patient told me that he really relies on these sensations. Another said that he even preferred to suffer from mild autonomic dysreflexia, just to have the feedback from his body. Yet another patient told me the same thing about neuropathic pain.

A final comment by a co-worker of mine, who is tetraplegic, deserves special mention as a conclusion. $\mathrm{He}$ said that when someone puts their hand on his shoulder while talking to him, it is a very comforting feeling. It is, therefore, not only through the magical medium of the scrub brush that we can minimize the negative effects of sensory deprivation, particularly for people with acute spinal cord injury.

\section{Dedication}

To the spinal cord patients and staff at BC Rehab., Vancouver, Canada.

\section{References}

1 Krishnan KR, Glass CA, Turner SM, Watt JW, Fraser MH Perceptual deprivation in the acute phase of spinal injury rehabilitation [comment]. Journal of the American Paraplegia Society 1992; 15(2): 60 65. Comment on: Kubzanski PE. In: The manipulation of human behavior. Biderman AD, Zimmer H, editors. New York: Wiley, 1961:51.

2 Richards JS, Hirt M. Melamed L. Spinal cord injury: a sensory restriction perspective. Archives of Physical Medicine and Rehabilitation 1982; 63: $195,199$.

3 Slade PD. Sensory deprivation and clinical psychiatry [comment]. British Journal of Hospital Medicine 1984; Nov: 256 260. Comment on: Zubek JP. In: Sensory deprivation: fifteen years of research. New York: Appleton Century-Crofts, 1969.

4 Goodman AL. Neurophysiological and psychopharmacological approaches to sensory deprivation phenomena [comment]. Progress in Neuro-psychopharmacology \& Biological Psichiatry 1982; 6: 95 110. Comments on:

a Archives of General Psychiatry 1964; 11: 255 - 276.

b Heron W. Cognitive and physiological effects of perceptual isolation. In: Solomon P, Kubzansky PE, Leiderman PH, Mendelson JH, Trumbell R, Wexler D editors. Sensor Deprivation Cambridge, Harvard University Press, 1961: 6-33.

c Perceptual and Motor Skills 1966; 22: 447-459.

d Aerospace Medicine 1971; 42: $840-842$.

e Archives of General Psychiatry 1960; 2: 147-155.

f Archives of General Psychiatry 1966; 14: $356-365$.

5 Bolin RH. Sensory deprivation: an overview. Nursing Forum 1974; 13(2): $241-258$.

6 Suedfield P. The benefits of boredom: sensory deprivation reconsidered. American Scientist 1975; 63: 60-69.

7 Zubeck JP, MacNeill M. Effects of immobilization: Behavioral and EEG changes. Candadian Journal of Psychology 1966; 20(3): $316-336$.

8 Davis JM, McCourt WF, Courtney J, Solomon P. Sensory deprivation. The role of social isolation. Archives of General Psychiatry 1961; 5: 106-112.

9 Hodes R. Electrocortical synchronization resulting from reduced proprioceptive drive caused by neuromuscular blocking agents [comment]. Electroencephalography and Clinical Neurophysiology 1962; 14: 220 - 232. Comment on: Sechenov IM. Reflexes of the brain. (1863). In: I. Sechenov, Selected Works. English translation, Moscow-Leningrad: State Publishing House for Biological and Medical Literature, 1935:336.
10 Richards JS, Hirt M, Melamed L. Spinal cord injury: a sensory restriction perspective [comment]. Archives of Physical Medicine and Rehabilitation 1982; 63: 195-199. Comment on: Hebb DO. Problem of consciouness and introspection. In: Delafresnaye JF editor. Brain Mechanisms and Consciousness. Springfield: Thomas, 1954: 402-21.

11 Solomon P, Leiderman PH, Mendelson J, Wexler D. Sensory deprivation: a review [comment]. American Journal of Psychiatry 1957; 114: 357 - 363. Comment on: Psychiatric Research Reports 1956; $5: 1$

12 Barr ML. Kiernan JA. The Human Nervous System. An Anatomical Viewpoint. 4th edition. Philadelphia: Harper and Row 1983.

13 Somjen G. Neurophysiology - the esssentials. Baltimore: Williams and Wilkins, 1983

14 Adey WR. Bors E. Porter RW. EEG sleep patterns after high cervical lesions in man. Archives of Neurology 1968; 19: 377 -. 383.

15 Hester GA. Effects of functional transection of the spinal cord on task performance under varied motivational conditions. Psichophysiology 1971; 8(4): 451-461.

16 Hester GA. Effects of functional transection of the spinal cord on task performance under varied motivational conditions [comment]. Psychophysiology 1971; 8(4): 451 -61. Comment on:

a Lindsley DB. Emotion. In: Stevens SS, editor. Handbook of Experimental Psychology. New York: Wiley, 1951; 473-516.

b Psychological Review 1955; 62: 243-254.

c Psychological Bulletin 1965; 64: 225-234.

17 Richards JS, Seitz MR, Eisele WA. Auditory processing in spinal cord injury: A preliminary investigation from a sensory deprivation perspective. Archives of Physical Medicine and Rehabilitation 1986; 67: 115-117.

18 Hester GA. Effects of functional transection of the spinal cord on task performance under varied motivational conditions [comment]. Psychophysiology 1971; 8(4): 451 461. Comment on:

a Psychological Review 1964; 71: 457-472.

b Hilgard E, Bower G. Theories of Learning. Third edition. New York: Appleton-Century-Crofts 1966

19 Richards JS, Seitz MR, Eisele WA. Auditory processing in spinal cord injury: A preliminary investigation from a sensory deprivation perspective [comment]. Archives of Physical Medicine and Rehabilitation 1986; 67: 115-17. Comment on: Rehabil. Psichol 1982; 27: 87-96.

20 Krishnan KR, Glass CA, Turner SM, Watt JW, Fraser MH. Perceptual deprivation in the acute phase of spinal injury rehabilitation. Journal of the American Paraplegia Society 1992; 15(2): $60-65$.

21 Bolin RH. Sensory deprivation: An overview [comment]. Nursing Forum 1974; 13(3): 241 - 58. Comment on:

a Nursing Research 1971; 20: 49.

b Archives of Internal Medicine 1972; 130: 225-226.

22 Lindsley D. Common factors in sensory deprivation, sensory distortion, and sensory overload. In: Solomon $\mathrm{P}$ (editor). Sensory Deprivation Cambridge: Harvard University Press, 1961: $174-194$

23 Zubeck JP. Counteracting effects of physical exercises performed during prolonged perceptual deprivation. Science 1963; 142: $504-506$

24 Gerhart KA. Increasing sensory and motor stimulation for the patient with quadriplegia. Physical Therapy 1979; 59(12): 1518 1520

25 Melzack R, Loesser JD. Phantom body pain in paraplegics: evidence for a central 'pattern generating mechanism' for pain. Pain 1978; 4(3): $195-210$.

26 Nicolelis MA, Lin RC, Woodward DJ, Chapin JK. Induction of immediate spatiotemporal changes in thalamic networks by peripheral block of ascending cutaneous information. Nature 1993: 361: $533-536$.

27 Volgyi B, Farkas T, Toldi J. Compensation of a sensory deficit inflicted upon newborn and adult animals. A behavioral study. Neuroreport 1993; 4: $827-29$.

28 Mazziotta JC, Phelps ME, Carson RE, Kuhl DE. Tomographic mapping of human cerebral metabolism: Sensory deprivation. Annals of Neurology 1982; 12: 435 - 444. 